\title{
Emittance and phase space exchange for advanced beam manipulation and diagnostics
}

\author{
Dao Xiang and Alex Chao \\ SLAC National Accelerator Laboratory, Menlo Park, California 94025, USA
}

(Received 21 May 2011; published 14 November 2011)

\begin{abstract}
Alternative chicane-type beam lines are proposed for exact emittance exchange between transverse phase space $\left(x, x^{\prime}\right)$ and longitudinal phase space $(z, \delta)$, where $x$ is the transverse position, $x^{\prime}$ is the transverse divergence, and $z$ and $\delta$ are relative longitudinal position and energy deviation with respect to the reference particle. Methods to achieve exact phase space exchanges, i.e., mapping $x$ to $z, x^{\prime}$ to $\delta, z$ to $x$, and $\delta$ to $x^{\prime}$, are suggested. Schemes to mitigate and completely compensate for the thick-lens effect of the transverse cavity on emittance exchange are studied. Some applications of the phase space exchange for advanced beam manipulation and diagnostics are discussed.
\end{abstract}

DOI: 10.1103/PhysRevSTAB.14.114001

\section{INTRODUCTION}

The ability to tailor a beam's $6 \mathrm{D}$ phase space is critical to meet the demands of various applications. For instance, imprinting a spatial chirp in beam with transverse cavities (also called crab cavities) can reduce the effective beam sizes at collision and improve the luminosity of colliders [1]. In emittance compensation [2,3], a solenoid is used to align the transverse phase ellipses of each beam slice to the same slope so that the projected emittance is minimized. In beam conditioning [4,5], particles with large betatron amplitudes are given extra energy to compensate for the path length differences from betatron oscillation, which may greatly enhance the gain in free electron lasers (FELs). In wakefield acceleration, an electron beam with a linearly ramped current profile can increase the transformer ratio [6].

Recently emittance exchange (EEX) between the transverse and longitudinal planes has been proposed to improve the FEL performance $[7,8]$. It is shown that, by exchanging the emittance in the transverse plane with that in the longitudinal plane, one can obtain a very small transverse emittance beam, taking advantage of the small longitudinal emittance from a photoinjector [8]. In addition to exchanging the emittance values, EEX can also be used to tailor the final longitudinal (transverse) phase spaces by shaping the initial transverse (longitudinal) phase spaces, because in EEX the longitudinal and transverse degrees of freedom are exchanged. For instance, EEX has been used to generate sub-ps bunch trains by modulating the initial transverse distribution with multislits [9].

The first beam line proposed for EEX consists of a simple four-dipole chicane and an rf transverse cavity [7]. While it is easy to implement this scheme, the EEX

Published by the American Physical Society under the terms of the Creative Commons Attribution 3.0 License. Further distribution of this work must maintain attribution to the author(s) and the published article's title, journal citation, and DOI.
PACS numbers: 29.27.Eg, 29.27.Bd, 29.27.Fh, 41.60.Cr

is not complete. An exact EEX beam line using two identical doglegs was later found [8]. While this beam line provides exact EEX, it also introduces offset in beam trajectory, which might be undesirable in some cases. In this paper, we propose a new chicane-type beam line to achieve exact EEX. We will show that by adding two quadrupoles in the center of the chicane, exact EEX can be achieved with a chicane. The thick-lens effect of the transverse cavity in EEX is systematically studied, and schemes to mitigate and completely compensate for this effect are also discussed. Simple methods to achieve phase space exchange (PSEX) in which the emittance values as well as the coordinates are exchanged, i.e., mapping $x$ to $z$, $x^{\prime}$ to $\delta, z$ to $x$, and $\delta$ to $x^{\prime}$, are suggested. Some applications of the PSEX, including generation of optical microbunches, measuring longitudinal-transverse correlation, etc., are discussed. We anticipate that PSEX may open up many new opportunities for advanced beam manipulation and diagnostics.

\section{EMITTANCE EXCHANGE}

Consider a planar lattice that allows coupling between the horizontal and longitudinal. Hereafter we will only consider the dynamics in the $x-z$ plane and neglect the uncoupled motion in vertical plane. Let the coordinate vector be $\left(x, x^{\prime}, z, \delta\right)^{T}$. Consider a transport section whose transport matrix from entrance to exit is

$$
\left[\begin{array}{ll}
A & B \\
C & D
\end{array}\right]
$$

where $A, B, C$, and $D$ are $2 \times 2$ matrices. Analysis shows that exact EEX is achieved when the elements in $A$ and $D$ are all zeros [7]. This special transport section has the property that it cleanly exchanges the $\left(x, x^{\prime}\right)$ and the $(z, \delta)$ degrees of freedom.

The first beam line [7] proposed by Cornacchia and Emma for EEX is shown in Fig. 1. It consists of a simple four-dipole chicane and a transverse cavity. 


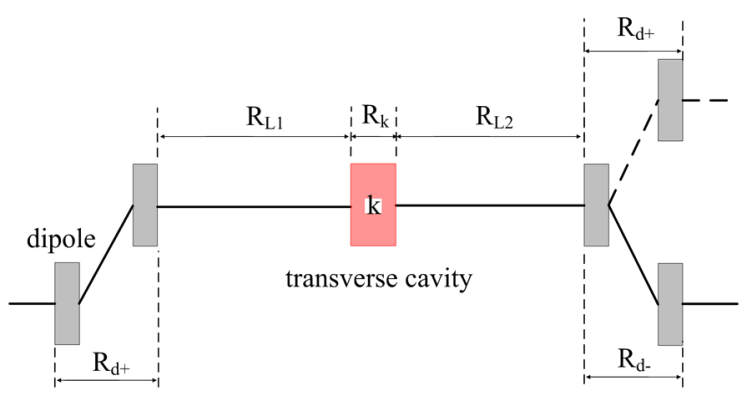

FIG. 1. Emittance exchange beam line based on a chicane and two doglegs.

The transfer matrix for the beam line based on a chicane is $R_{1}=R_{d-} R_{L_{2}} R_{k} R_{L_{1}} R_{d+}$, where $R_{d+}$ and $R_{d-}$ are the transfer matrices for the first and the second half of the chicane, $R_{L_{1}}$ and $R_{L_{2}}$ are the transfer matrices for the drifts before and after the transverse cavity, and $R_{k}$ is the transverse cavity transfer matrix. The transfer matrices for these elements can be found in [7]. Partial EEX is achieved when $\eta k=1$, where $\eta$ is the dispersion in the center of the chicane and $k=2 \pi e V / \lambda E$ is the dimensionless deflection strength of the transverse cavity, $V$ is the deflection voltage, $\lambda$ is the wavelength of the rf field, and $E$ is the beam energy. However, the EEX is not complete in this beam line because there are nonzero elements in $A$ and $D$.

An exact exchange optics was later found by Kim [8]. Instead of a chicane, the transverse cavity is put between two identical doglegs, as shown with the dashed line in Fig. 1. The transfer matrix for this beam line is $R_{2}=$ $R_{d+} R_{L_{2}} R_{k} R_{L_{1}} R_{d+}$. Exact EEX is achieved when $\eta k=$ -1 . Based on this optics, experiments at FNAL [10] have been performed and others are being planned at ANL [11]. EEX using a TEM10 mode laser, instead of rf transverse cavity, was also suggested [12] and shown to be promising in obtaining an ultrasmall transverse emittance beam.

An apparent difference between the Cornacchia-Emma beam line and the Kim beam line is that the former is mirror symmetric with respect to the transverse cavity while the latter is not. This observation leads us to conjecture that a beam line with mirror symmetry, while being simple, cannot provide exact EEX. We will prove this below. For simplicity, we will use a thin-lens matrix for the transverse cavity, and further limit our analysis on using dipoles, transverse cavities, quadrupoles, and other conventional elements for EEX.

Following the analysis in [13] and assuming no additional $\mathrm{rf}$ cavities other than the transverse cavity is used in the beam line, the transfer matrix of the beam line upstream of the transverse cavity may be written as

$$
M=\left[\begin{array}{cccc}
a & b & 0 & \eta \\
c & d & 0 & \eta^{\prime} \\
c \eta-a \eta^{\prime} & d \eta-b \eta^{\prime} & 1 & \xi \\
0 & 0 & 0 & 1
\end{array}\right]
$$

where $|M|=a d-b c=1$. Assuming the beam line downstream of the transverse cavity is the mirror image of the upstream beam line with respect to the transverse cavity, its transfer matrix $M_{m}$ is related to $M$ as [14]

$$
M_{m} U M=U,
$$

where $U$ is the " $U$-turn" matrix,

$$
U=\left[\begin{array}{cccc}
1 & 0 & 0 & 0 \\
0 & -1 & 0 & 0 \\
0 & 0 & -1 & 0 \\
0 & 0 & 0 & 1
\end{array}\right]
$$

Note $M_{m}$ equals to the transfer matrix of beam line $M$ if the particle goes through it with a reversed direction. The physical meaning of Eq. (3) and the $U$-turn matrix is that after one particle passes through the beam line $M$, if it makes a $U$ turn such that its direction of motion is reversed, it will return to its initial position at the entrance to the beam line $M$ with its momentum reversed. It is straightforward to find that the transfer matrix $M_{m}$ is

$M_{m}=U M^{-1} U^{-1}=\left[\begin{array}{cccc}d & b & 0 & b \eta^{\prime}-d \eta \\ c & a & 0 & a \eta^{\prime}-c \eta \\ -\eta^{\prime} & -\eta & 1 & \xi \\ 0 & 0 & 0 & 1\end{array}\right]$.

Then we found the transfer matrix for a beam line with mirror symmetry and with a transverse cavity in the center to be

$$
R_{M}=M_{m} R_{k} M=\left[\begin{array}{cccc}
a d+b c-\eta k & 2 b d & b k & -d \eta^{2} k+b \zeta^{+} \\
2 a c & a d+b c+\eta k & a k & -c \eta^{2} k+a \zeta^{+} \\
-c \eta^{2} k+a \zeta^{-} & -d \eta^{2} k+b \zeta^{-} & 1-\eta k & 2 \xi-2 \eta \eta^{\prime} \\
a k & b k & 0 & 1+\eta k
\end{array}\right]
$$

where $\zeta^{-}=\eta^{\prime}(\eta k-2)+k \xi$ and $\zeta^{+}=\eta^{\prime}(\eta k+2)+k \xi$. It is obvious that several elements of the transfer matrix in $A$ and $D$ (for instance, $R_{11}$ and $R_{22}, R_{33}$ and $R_{44}$ ) cannot be zero simultaneously and exact EEX cannot be achieved. 


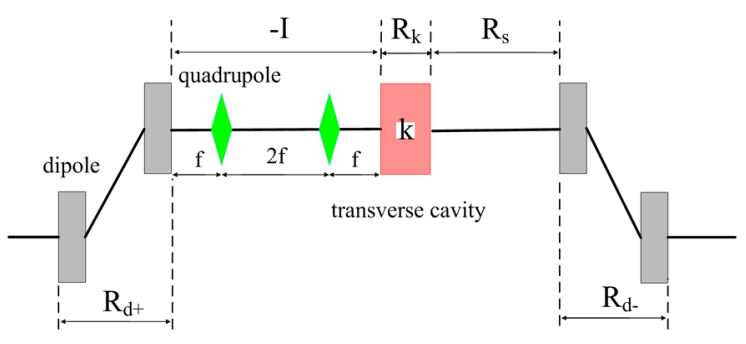

FIG. 2. A chicane-type exact EEX beam line. Two quadrupoles (green diamonds) are put upstream of the transverse cavity to reverse the dispersion.

\section{EEX WITH A CHICANE-TYPE BEAM LINE}

From a practical point of view, EEX with a chicane may be more desirable because of its simplicity, wide availability, and minimal perturbation to existing beam lines (e.g. it does not introduce offset in beam trajectory and turning off the transverse cavity allows dispersion to return to zero). But it has a lower performance compared to the two-dogleg scheme. Here we show that the chicane scheme can achieve exact EEX as well by adding (at least two) quadrupoles (the mirror symmetry is broken too). The scheme is shown in Fig. 2.

Two quadrupoles together with three drifts are used to form a negative unity transfer matrix for the transverse plane. The focal lengths of the two quadrupoles are both $f$. With the lengths of the drifts chosen as shown in Fig. 2, the $4 \times 4$ transfer matrix for the $-I$ section is

$$
R_{-I}=\left[\begin{array}{cc}
-I & 0 \\
0 & I
\end{array}\right]
$$

where

$$
I=\left[\begin{array}{ll}
1 & 0 \\
0 & 1
\end{array}\right]
$$

is the $2 \times 2$ unity matrix. The negative unity section reverses the dispersion of the first half of the chicane, which is optically equivalent to flipping the sign of a dogleg. The transfer matrix for the whole beam line $\left(R_{c}=\right.$ $\left.R_{d^{-}} R_{S} R_{k} R_{-I} R_{d+}\right)$ when $\eta k=1$ is

$$
R_{c}=\left[\begin{array}{cccc}
0 & 0 & k(L+S) & k \xi(L+S)-\eta \\
0 & 0 & k & k \xi \\
-k \xi & \eta-k L \xi & 0 & 0 \\
-k & -k L & 0 & 0
\end{array}\right]
$$

where $L, \xi$, and $\eta$ are the length, the momentum compaction, and dispersion of the first half of the chicane. Equation (7) implies that such a beam line also provides exact exchange for the transverse and longitudinal emittances. Further analysis shows that the angular to spatial element for the transfer matrix $R_{-I}$ does not need to be zero for an exact exchange, which indicates that the distance between the negative unity matrix beam line and the transverse cavity can be arbitrary. Also the $R_{-I}$ section can be put downstream of the transverse cavity, for which case exact EEX is achieved with $\eta k=-1$. It should be pointed out that, if we take the dispersion at the center of the transverse cavity as $\eta^{*}$, the condition to achieve exact EEX is always $\eta^{*} k=-1$ (for the beam line in Fig. 2 we have $\left.\eta^{*}=-\eta\right)$ [13].

\section{THICK-LENS EFFECTS}

In the analysis above, we have used a thin-lens matrix for the transverse cavity where the energy kick and angular kick is instantaneous and the length of the cavity is neglected. When the finite length of the transverse cavity is taken into account, the thick-lens transfer matrix of a transverse cavity with a given dimensionless kick strength $k$ in the infinite number of cells limit is found to be $[7,13]$

$$
R_{k, \text { thick }}=\left[\begin{array}{cccc}
1 & L_{c} & k L_{c} / 2 & 0 \\
0 & 1 & k & 0 \\
0 & 0 & 1 & 0 \\
k & k L_{c} / 2 & k^{2} L_{c} / 6 & 1
\end{array}\right]
$$

where $L_{c}$ is the length of the cavity. The corresponding transfer matrix for the chicane-type beam line with the thick-lens effect included is

$$
R_{c, \text { thick }}=\left[\begin{array}{cccc}
0 & -L_{c} / 6 & k\left(L+S+L_{c} / 3\right) & -\eta+k \xi\left(L+S+L_{c} / 3\right) \\
0 & 0 & k & k \xi \\
-k \xi & \eta-k\left(L+L_{c} / 3\right) \xi & k^{2} L_{c} \xi / 6 & k^{2} L_{c} \xi^{2} / 6 \\
-k & -k\left(L+L_{c} / 3\right) & k^{2} L_{c} / 6 & k^{2} L_{c} \xi / 6
\end{array}\right]
$$

Because of the nonzero elements in the submatrices $A$ and $D, \mathrm{EEX}$ is incomplete. This is also true for the twodogleg scheme. For this case, the final projected emittances after EEX beam line become $\epsilon_{x, z}^{2}=\epsilon_{z 0, x 0}^{2}+\frac{\epsilon_{x 0} \epsilon_{z 0} k^{2} L_{c}^{2} \gamma_{x 0}}{36 \beta_{z 0}}\left[\xi^{2}+\left(\beta_{z 0}-\alpha_{z 0} \xi\right)^{2}\right]$,

where $\epsilon_{z 0, x 0}$ is the initial longitudinal (transverse) emittance, $\alpha, \beta$, and $\gamma$ are the Twiss parameters. 
In the thin-lens approximation, the transverse cavity only introduces $x^{\prime}-z$ and $x-\delta$ correlation. When the finite length is taken into account, it also introduces $x^{\prime}-x$, $x^{\prime}-\delta, z-\delta$, and $z-x$ correlations that lead to nonexact EEX. Intuitively, one can shape the initial phase space to reduce the values of $x^{\prime}$ and $z$ at the cavity to mitigate the degradations from these additional correlations. For instance, by minimizing $\gamma_{x 0}$ and letting $\alpha_{z 0}=\beta_{z 0} / \xi$, the beam divergence and bunch length at the transverse cavity can be minimized, which also minimizes the degradation from the finite length of the cavity.

Another way to mitigate the thick-lens effect may be to reduce the strength and length of the cavity, as can be seen in the second term in Eq. (10) that the incomplete EEX term scales as $k^{2} L_{c}^{2}$. However, because the condition to achieve EEX in the beam lines discussed above is $|\eta k|=1$, it appears that the dispersion of the dogleg needs to be accordingly increased when the strength of the cavity is reduced. We will show in Sec. V that it is also possible to use a weaker cavity to achieve EEX without increasing the dispersion of the dogleg.

In addition to mitigating the thick-lens effect by shaping the initial phase space and using a weaker transverse cavity, complete compensation for the effect to allow exact EEX is also possible. Analysis shows that, while the thick-lens transfer matrix has nonzero $R_{12}, R_{13}, R_{42}$, and $R_{43}$ elements compared to the thin-lens case, the incomplete EEX is solely caused by the $R_{43}$ term. Using a simple rf cavity in the fundamental mode with $R_{43}=-k^{2} L_{c} / 6$ to cancel the longitudinal acceleration in the transverse cavity $\left[R_{43}\right.$ in Eq. (8)] allows, to the first order, exact EEX when the thicklens effect of the transverse cavity is taken into account [15].

\section{EEX WITH $\left|\boldsymbol{\eta}^{*} / \boldsymbol{\eta}\right| \neq 1$}

In all the beam lines discussed above, EEX requires $|\eta k|=1$, where $\eta$ is the dispersion of the dogleg. This condition appears to make EEX difficult to apply for high energy beams where either a huge dogleg with very large $\eta$ or a long transverse cavity with very high voltage is needed to satisfy the condition. For instance, assuming a dogleg with $\eta=0.5 \mathrm{~m}$, an $\mathrm{x}$-band cavity with $V=84 \mathrm{MV}$ is needed for $10 \mathrm{GeV}$ beam to satisfy the condition $|\eta k|=1$. Take the $\mathrm{x}$-band transverse cavity [16] to be used for measurements of electron beam and x-ray pulse temporal profiles in LCLS as an example, assuming an input power of $30 \mathrm{MW}$, a $3.2 \mathrm{~m}$ long structure is needed to provide $84 \mathrm{MV}$ kick. This long cavity length may result in serious thick-lens effects in EEX.

It is worth mentioning that it is the dispersion at the center of the transverse cavity $\eta^{*}$ rather than the dispersion generated by the first dogleg $\eta$ that needs to match the strength of the transverse cavity [13]. Here we want to point out that, by adding telescope beam lines before and after the transverse cavity, we can change the ratio of $\eta^{*} / \eta$ and the condition $|\eta k|=1$ can be relaxed.
As shown in Fig. 3, two focusing quadrupoles with focal length $f_{1}$ and $f_{2}$ (green diamonds) are put upstream of the transverse cavity to form a telescope beam line that magnifies the beam with a magnification ratio $N=f_{2} / f_{1}$, which also increases the local dispersion in the transverse cavity to $\eta^{*}=-N \eta$. The transfer matrix is

$$
\left[\begin{array}{cc}
R_{-N} & 0 \\
0 & I
\end{array}\right],
$$

where

$$
R_{-N}=\left[\begin{array}{cc}
-N & a \\
0 & -1 / N
\end{array}\right]
$$

After the transverse cavity, one focusing quadrupole with focal length $f_{2}$ and one defocusing quadrupole (blue diamond) with focal length $f_{1}$ are used to restore the beam to its initial size. The transfer matrix for this section is

$$
\left[\begin{array}{cc}
R_{1 / N} & 0 \\
0 & I
\end{array}\right],
$$

where

$$
R_{1 / N}=\left[\begin{array}{cc}
1 / N & b \\
0 & N
\end{array}\right]
$$

In $R_{-N}$ and $R_{1 / N}, a$ and $b$ depend on the drift lengths before and after the quadrupoles, but they do not affect the condition to achieve exact EEX. It is straightforward to prove that exact EEX for such a beam line is achieved when $\eta k=1 / N$.

Note the second term in Eq. (10) scales as $k^{2} L_{c}^{2} \sim N^{-4}$, therefore by having $N>1$, shorter transverse cavity with lower deflection voltage can be used in EEX which may greatly mitigate the thick-lens effects. On the other hand, for given deflection voltage and dispersion of the dogleg, EEX can be implemented at higher beam energy.

Take the LCLS x-band transverse cavity as an example, its aperture has a diameter of $10 \mathrm{~mm}$ [16]. Assuming $50 \mathrm{~m}$ beta function at the entrance to the telescope beam line and $1 \mu \mathrm{m}$ transverse emittance (corresponding beam size is $50 \mu \mathrm{m}$ before the telescope beam line), a telescope beam line with $N=10$ may be used to reduce the required cavity length to $0.32 \mathrm{~m}$ for EEX. The beam size after the telescope beam line is accordingly increased to about $0.5 \mathrm{~mm}$ which

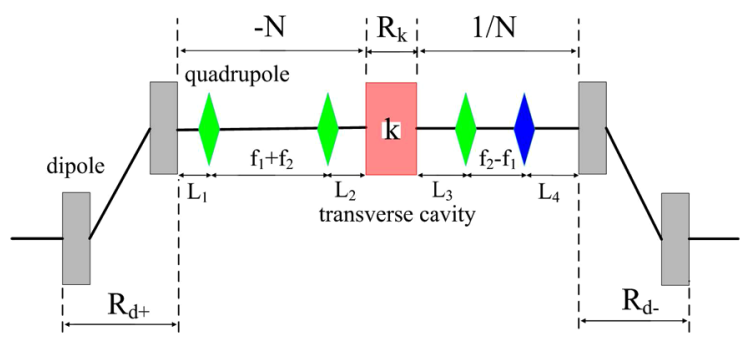

FIG. 3. Exact EEX beam line with $|\eta k|<1$. 
TABLE I. Main beam and EEX beam line parameters at NLCTA.

\begin{tabular}{lc}
\hline \hline Electron beam energy & $60 \mathrm{MeV}$ \\
Electron beam charge & $20 \mathrm{pC}$ \\
Normalized horizontal emittance & $3.00 \mathrm{~mm} \mathrm{mrad}$ \\
Normalized longitudinal emittance & $0.300 \mathrm{~mm} \mathrm{mrad}$ \\
Initial rms bunch length & $100 \mu \mathrm{m}$ \\
Dipole length & $0.2 \mathrm{~m}$ \\
Distance between the 1st and 2nd dipoles & $1.175 \mathrm{~m}$ \\
Bending angle of the dipoles & $9.56 \mathrm{deg}$ \\
Momentum compaction of the dogleg & $36.6 \mathrm{~mm}$ \\
Dispersion of the dogleg & $0.23 \mathrm{~m}$ \\
Length of the transverse cavity & $0.1 \mathrm{~m}$ \\
\hline \hline
\end{tabular}

is still much smaller than the aperture of the cavity. This may significantly mitigate the thick-lens effect.

It should be emphasized that, while using a telescope to increase the local dispersion at the cavity does not increase the offset of the beam trajectory before and after the dogleg, it does increase the transverse beam size in the transverse cavity, as discussed above. Depending on specific parameters, the aperture of the transverse cavity may accordingly need to be increased, which may reduce the shunt impedance of the cavity and thus require more power to provide a given dimensionless kick strength. This may set a constraint on the magnification ratio $N$ beyond which the disadvantage may outweigh the gain. Nevertheless, even if the required power may need to be increased, the dimensionless kick strength of the cavity required in EEX is still reduced when $N>1$, which will mitigate the thicklens effect too. Therefore, we believe adding telescope beam line to vary the ratio of $\eta^{*} / \eta$ may provide more flexibility in implementing EEX.

\section{REPRESENTATIVE SIMULATION RESULTS}

A chicane integrated with 12 quadrupoles is available at SLAC's Next Linear Collider Test Accelerator (NLCTA [17]). An 11-cell x-band (frequency at $11.424 \mathrm{GHz}$ ) transverse cavity, originally installed for heating the beam slice energy spread to facilitate demonstration of the echoenabled harmonic generation technique [18], is also available. Three of the quadrupoles between the second and third dipoles can be used to form the $-I$ section, and relocating the transverse cavity to the center of the chicane allows the chicane-type EEX beam line to be tested at NLCTA.

The performance of the EEX with the chicane-type beam line at NLCTA has been simulated with ELEGANT [19] including the thick-lens effect and second order optics effects. The main parameters for the chicane-type EEX beam line and the electron beam at the entrance to the EEX beam line are listed in Table I. The beam phase spaces before and after the EEX beam line are shown in Fig. 4.

The initial transverse and longitudinal emittances are $\epsilon_{n, x 0}=3.00 \mu \mathrm{m}, \epsilon_{n, z 0}=0.300 \mu \mathrm{m}$ and the final emittance after exchange is $\epsilon_{n, x 1}=0.309 \mu \mathrm{m}, \epsilon_{n, z 1}=$ $3.01 \mu \mathrm{m}$. The growth in the final horizontal emittances is mainly from thick-lens effect and second order effects. In a separate simulation which also takes into account the second order effects, the horizontal emittance reduces to $0.304 \mu \mathrm{m}$ when a thin transverse cavity $\left(L_{c}=0.01 \mathrm{~m}\right)$ is used. The horizontal emittance further reduces to
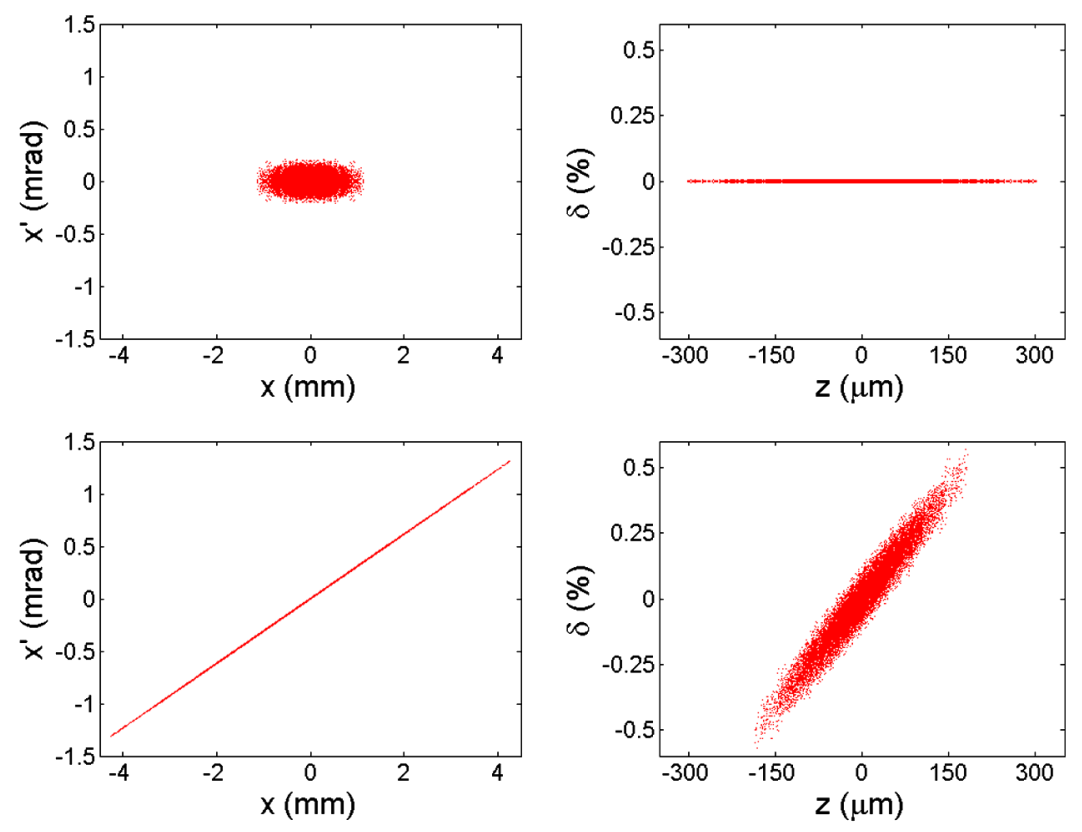

FIG. 4. Transverse phase space distribution (left plots) and longitudinal phase space distribution (right plots) before (top row) and after (bottom row) the chicane-type EEX beam line. 
$0.3002 \mu \mathrm{m}$ if the particles are tracked only to the first order.

We also simulated the EEX with $\left|\eta^{*} / \eta\right| \neq 1$. Very interestingly, when the telescope beam line is used with $N=3$, the deflection voltage is reduced by a factor of 3 , which results in a final horizontal emittance of $\epsilon_{n, x 1}=$ $0.303 \mu \mathrm{m}$. Now the emittance growth is mainly from the second order effects.

\section{PHASE SPACE EXCHANGE}

It follows from Eq. (7) that $z_{f}=-k \xi x_{i}+(\eta-k L \xi) x_{i}^{\prime}$ and $\delta_{f}=-k x_{i}-k L x_{i}^{\prime}$. Therefore, by shaping the initial transverse phase space $\left(x_{i}, x_{i}^{\prime}\right)$, the final longitudinal phase space distribution $\left(z_{f}, \delta_{f}\right)$ can be tailored [20]. Note it is easy to shape the $x$ distribution using masks with various shapes, but shaping $x^{\prime}$ distribution can be technically challenging. In practice, one may make the final $z_{f}$ and $\delta_{f}$ dominated by initial $x_{i}$ rather than $x_{i}^{\prime}$, so that shaping $x$ distribution suffices. This is typically achieved by properly choosing the parameters for the EEX beam line and increasing the beam size at the entrance to the EEX beam line. Experiments at FNAL have demonstrated that one can generate sub-ps bunch train by modulating the initial transverse distribution with multislits [9]. However, the crossterm dependence of $z_{f}$ and $\delta_{f}$ on $x_{i}$ and $x_{i}^{\prime}$ may set a limit on how well the phase space can be tailored.

Here we show further that by adding more quadrupoles upstream and downstream of the EEX beam line, we can achieve exact phase space exchange (PSEX), i.e., mapping $x$ to $z, x^{\prime}$ to $\delta, z$ to $x$, and $\delta$ to $x^{\prime}$. This allows more advanced surgery for beam phase space manipulation.

For the system that provides exact EEX, its transfer matrix has the form

$$
\left[\begin{array}{ll}
0 & B \\
C & 0
\end{array}\right] \text {. }
$$

We now add a section consisting of drifts and quadrupoles in front, and another similar section after the exchanger beam line. Let these two sections' $4 \times 4$ transport matrices be

$$
\left[\begin{array}{ll}
E & 0 \\
0 & I
\end{array}\right]
$$

and

$$
\left[\begin{array}{ll}
F & 0 \\
0 & I
\end{array}\right]
$$

respectively, where $I$ is a $2 \times 2$ unit matrix and $E$ and $F$ are some other general $2 \times 2$ matrices with unit determinants.

The total transport matrix is then

$$
\left[\begin{array}{cc}
F & 0 \\
0 & I
\end{array}\right]\left[\begin{array}{ll}
0 & B \\
C & 0
\end{array}\right]\left[\begin{array}{cc}
E & 0 \\
0 & I
\end{array}\right]=\left[\begin{array}{cc}
0 & F B \\
C E & 0
\end{array}\right] .
$$

By lattice matching using the two drift-quadrupole sections, it is possible to create arbitrary results for $F B$ and $C E$ to meet the desired lattice properties. For example, if we design the two drift-quadrupole sections such that $F=$ $B^{-1}$ and $E=C^{-1}$, then we would have a transport line that has a transport matrix

$$
\left[\begin{array}{ll}
0 & I \\
I & 0
\end{array}\right]
$$

i.e. it will map $x$ to $z, x^{\prime}$ to $\delta, z$ to $x$, and $\delta$ to $x^{\prime}$. For this case, the final temporal profile of the bunch, to the first order, is a complete replica of the initial transverse profile, and the beam current may be arbitrarily shaped using transverse masks.

Take the two-dogleg beam line which has already been used to generate sub-ps bunch trains in FNAL as an example, such an exact PSEX requires

$$
E=\left[\begin{array}{cc}
-\left(L+L_{1}\right) / \eta & -\eta+\left(L+L_{1}\right) \xi / \eta \\
1 / \eta & -\xi / \eta
\end{array}\right]
$$

and

$$
F=\left[\begin{array}{cc}
-\xi / \eta & -\eta+\left(L+L_{2}\right) \xi / \eta \\
1 / \eta & -\left(L+L_{2}\right) / \eta
\end{array}\right] .
$$

In practice, four matching quadrupoles before and after the EEX beam line can be used to construct the beam line with a proper transfer matrix to achieve PSEX. It is worth pointing out that mapping $x$ to $\delta, x^{\prime}$ to $z$, and/or $z$ to $x^{\prime}$, and $\delta$ to $x$ are all also possible, but they will have opposite sign, i.e., when $x$ is mapped to $\delta, x^{\prime}$ will be mapped to $-z$. It is also straightforward to include magnification in PSEX in which case when $x$ is mapped to $N z, x^{\prime}$ is accordingly mapped to $\delta / N$.

\section{SOME APPLICATIONS OF PSEX}

Exact PSEX may have wide applications. For instance, by mapping $z$ exactly to $x$, the beam's temporal profile can be easily measured with a view screen. By mapping $x$ exactly to $z$, to the first order, one may generate electron beam with arbitrary current using proper transverse masks.

In this section we give several applications of PSEX. For simplicity we will add drifts and quadrupoles before and after the two-dogleg EEX beam line to achieve PSEX. PSEX based on the chicane-type beam line and other beam lines can be studied similarly.

\section{A. Generation of optical microbunches}

Using EEX to generate sub-ps microbunch trains with a transverse multislit has been experimentally demonstrated [9]. Note the cross-term dependence in EEX limited the duration of microbunches to $(\eta-k L \xi) \sigma_{x}^{\prime}$, regardless of how narrow the slit is. While this term does not significantly affect the generation of sub-ps microbunches, it may 


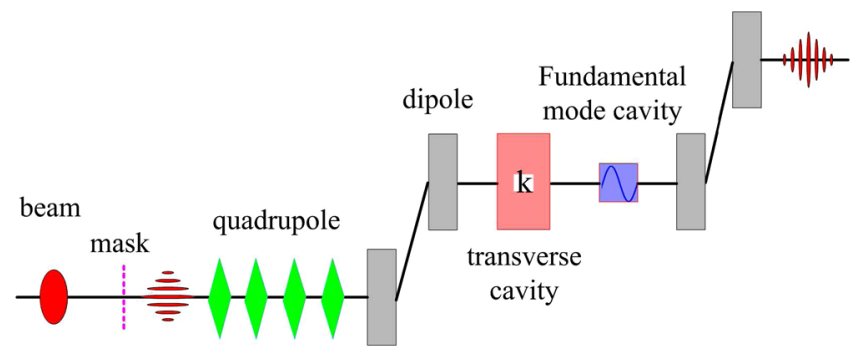

FIG. 5. Schematic of a beam line to generate optical microbunch based on PSEX.

limit the generation of microbunches with spacing in the optical wavelength and below.

For net acceleration in direct laser accelerator, the duration of the bunch needs to be much shorter than the laser wavelength [21]. Typically such a short bunch or bunch train is generated with the inverse FEL in which the beam is energy modulated by a laser and then a chicane is used to convert the energy modulation into density modulation so that a microbunch train equally separated by the laser wavelength is generated. We will show below that such kind of optical microbunches can be alternatively generated through PSEX.

In the example below, the parameters of the dipoles and the transverse cavity are the same as those in Table I, but the beam parameters are different. The beam energy is assumed to be $120 \mathrm{MeV}$ such that after PSEX, the optical microbunches can interact effectively with optical lasers with a conventional undulator. The longitudinal and transverse emittance are assumed to be both $0.3 \mathrm{~mm} \mathrm{mrad}$ so that after PSEX the emittance in each plane remains the same. The rms bunch length is assumed to be $30 \mu \mathrm{m}$ to mitigate the second order effect. The schematic layout of the beam line to generate optical microbunches is shown in Fig. 5. A mask is first used to generate density modulation in transverse direction. Four quadrupoles with effective lengths of $12.6 \mathrm{~cm}$ and strengths of $12.5551 \mathrm{~m}^{-2}$, $-9.7084 \mathrm{~m}^{-2},-11.2709 \mathrm{~m}^{-2}$, and $8.0711 \mathrm{~m}^{-2}$ are put upstream of the two-dogleg EEX beam line to provide the suitable transfer matrix required for mapping $x$ exactly to $z$ and $x^{\prime}$ to $\delta$ with a unity magnification ratio. The distances between the quadrupoles are all $1 \mathrm{~m}$. An $\mathrm{x}$ band transverse cavity with rf power of about $8 \mathrm{MW}$ is used to provide the required dimensionless kick strength of $4.35 \mathrm{~m}^{-1}$ for EEX. Furthermore, a short $(3 \mathrm{~cm})$ fundamental mode rf cavity with voltage of about $150 \mathrm{kV}$ is put after the transverse cavity to compensate for the thick-lens effect.

In our simulation we assume the transverse mask consists of a series of slits equally separated by $800 \mathrm{~nm}$ (typical wavelength of a Ti:sapphire laser) with full slit width of $200 \mathrm{~nm}$. In practice, such microslits may be made with nanofabrication techniques. For simplicity, we further assume only the particles that go through the slit can enter the PSEX beam line, while other particles that strike on the mask are completely stopped. In practice, the mask may not have the sufficient stopping power for high energy beam, and as a result the beam may penetrate through the mask. But for these particles their divergence will
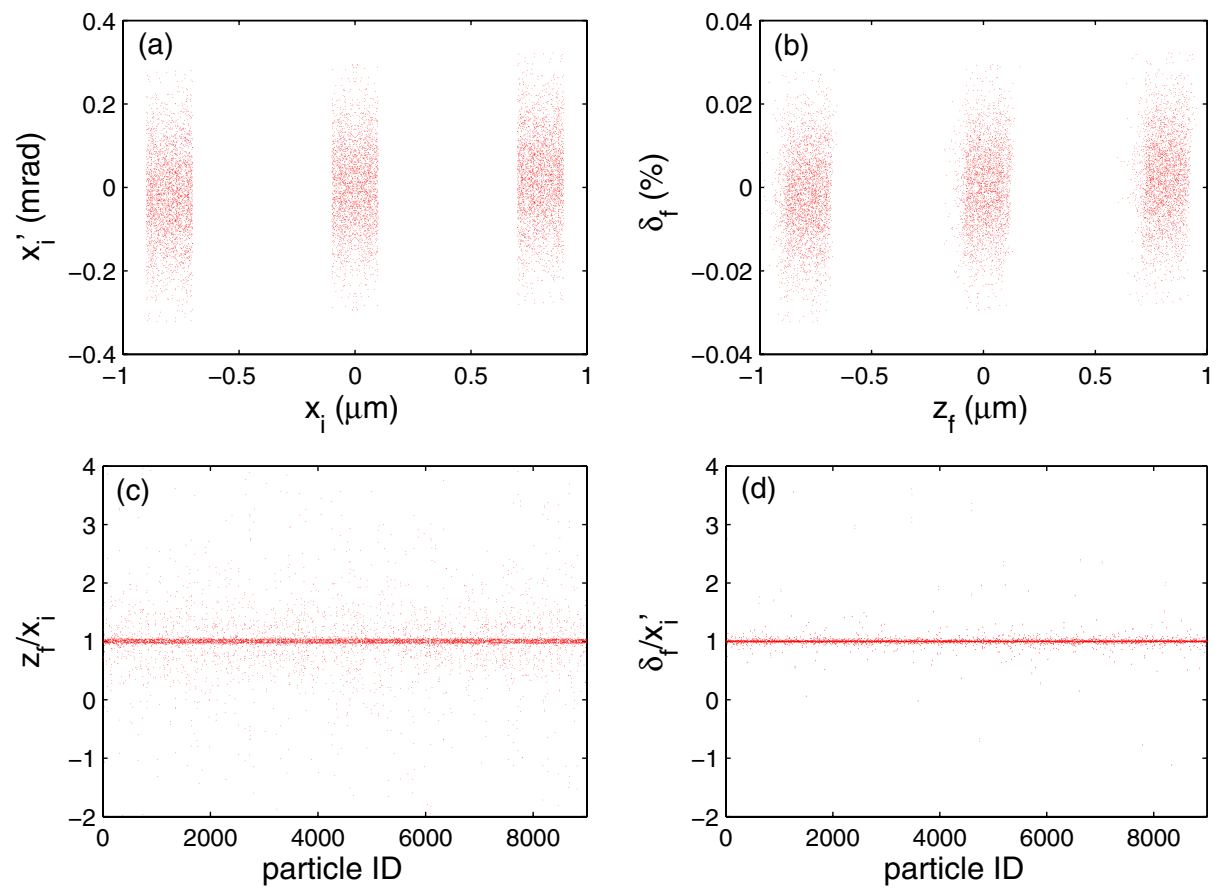

FIG. 6. (a) Initial transverse phase space after the mask. (b) Final longitudinal phase space after PSEX. (c) Ratio of $z_{f}$ to $x_{i}$. (d) Ratio of $\delta_{f}$ to $x_{i}^{\prime}$. 

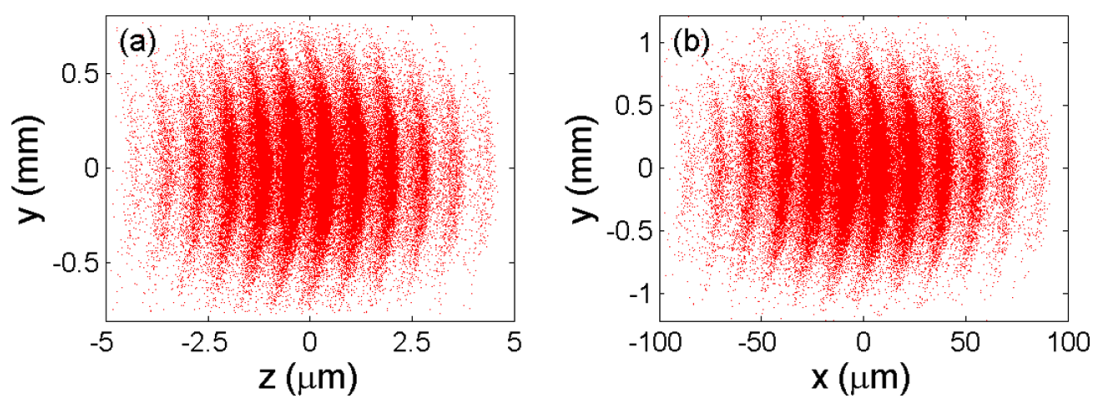

FIG. 7. (a) Initial $z-y$ correlation. (b) Final $x-y$ correlation after PSEX.

increase significantly from the Coulomb scattering and they should form a uniform background which in many cases does not greatly affect the performance of the microbunch train. The transverse distribution of the beam after the mask is shown in Fig. 6(a) (only part of the beam is shown). After the mask, the particles are tracked through the PSEX beam line using the code ELEGANT with second order effects taken into account. The final longitudinal phase space for the particles in Fig. 6(a) is shown in Fig. 6(b), where one can see that optical microbunches equally separated by $800 \mathrm{~nm}$ with full duration of about $200 \mathrm{~nm}$ are generated. Figures 6(c) and 6(d) show the ratio of the final longitudinal position $z_{f}$ to the initial transverse position $x_{i}$ and the final energy deviation $\delta_{f}$ to the initial divergence $x_{i}^{\prime}$ for each particle. It can be seen that near perfect PSEX is achieved with one-to-one mapping from $x$ to $z$ and $x^{\prime}$ to $\delta$. The deviation from unity in Figs. 6(c) and $6(d)$ is mainly from the second order effects.

It should be pointed out that it is straightforward to include magnification ratio to change the ratio $z_{f} / x_{i}$ to vary the spacing of the microbunch. This offers more flexibility as compared to the conventional method in which the spacing of the microbunch is determined by the laser wavelength. With higher energy and lower emittance beam, it might be even possible to generate microbunch in the nanometer and Angstrom regime, which may significantly reduce the required undulator length to reach FEL saturation [22].

\section{B. Measure $z-y$ correlation}

Recently a longitudinal-to-transverse mapping technique in which $z$ is exactly mapped to $x$ has been proposed to measure the temporal profile of an ultrashort bunch [23]. With PSEX, in addition to mapping $z$ to $x, \delta$ is also mapped to $x^{\prime}$, so it is straightforward to measure the temporal profile and the longitudinal phase space distribution $(z, \delta)$ by measuring the transverse phase space $\left(x, x^{\prime}\right)$ after PSEX. Here we consider another application of PSEX for measuring $z-y$ correlation in beam phase space.

It is well known that transverse emittance affects the FEL microbunching and gain. This can be understood as a smearing effect in which particles that have larger betatron amplitude have longer path lengths and tend to lag behind the reference particle. As a result, the "wave front" of the microbunch from FEL interaction is curved and the bunching is reduced. Very similarly for the optical microbunches generated from laser modulation, the wave front of the microbunch is curved and nonlinear correlation between the particle's longitudinal coordinate $z$ and the transverse coordinates $(x$ and $y$ ) is developed after the microbunch further propagates downstream, as shown in Fig. 7(a). Measurement of this correlation may be useful to understand the physics and achieve optimal performance. With PSEX, the initial $z-y$ correlation will be converted to $x-y$ correlation and it may be measured with a view screen.

For the distribution shown in Fig. 7(a), a PSEX beam line is used to map $z$ to $x$ with a magnification ratio 20 and the final $x-y$ distribution after PSEX is shown in Fig. 7(b). The beam line is similar to that shown in Fig. 5, except that four matching quadrupoles are put downstream of the twodogleg EEX beam line. Simulation with the ELEGANT code indicates that the PSEX technique is able to convert the initial nonlinear correlation in $z-y$ into $x-y$ correlation. This may provide more information about the beam phase space not accessible with other conventional diagnostic techniques.

It should be pointed out that in our studies we focused on single-particle dynamics, and the collective effects such as coherent synchrotron radiation (CSR), space charge effect, etc. were not included. In practical cases, the CSR effect can be mitigated using weak bends, and it is also possible to significantly suppress the CSR effect with small-gap vacuum chambers [24]. The space charge effect typically does not play an important role when beam energy is high. Nevertheless, these collective effects may limit the application of EEX and PSEX to high peak current beam and degrade their performance. However, detailed evaluation of their effects on specific application with varying beam parameters is beyond the scope of this paper.

\section{CONCLUSIONS}

In this paper alternative chicane-type beam lines are proposed for exact EEX between horizontal phase space $\left(x, x^{\prime}\right)$ and longitudinal phase space $(z, \delta)$. By adding drifts and quadrupoles before and after the EEX beam line, it is shown that exact PSEX, i.e., mapping $x$ to $z, x^{\prime}$ to $\delta, z$ to $x$, 
and $\delta$ to $x^{\prime}$, can also be achieved. Some applications of the PSEX, including generation of optical microbunches, measuring $z-y$ correlation, etc. are discussed. We anticipate that PSEX may open up many new opportunities for advanced beam manipulation and diagnostics.

\section{ACKNOWLEDGMENTS}

This work was supported by the U.S. DOE under Contract No. DE-AC02-76SF00515.

[1] R. B. Palmer, Report No. SLAC-PUB-4707, 1988.

[2] B. E. Carlsten, Nucl. Instrum. Methods Phys. Res., Sect. A 285, 313 (1989).

[3] L. Serafini and J. B. Rosenzweig, Phys. Rev. E 55, 7565 (1997).

[4] A. M. Sessler, D. H. Whittum, and L.-H. Yu, Phys. Rev. Lett. 68, 309 (1992).

[5] A. Wolski, G. Penn, A. Sessler, and J. Wurtele, Phys. Rev. ST Accel. Beams 7, 080701 (2004).

[6] P. Chen, J. Su, J. Dawson, K. Bane, and P. Wilson, Phys. Rev. Lett. 56, 1252 (1986).

[7] M. Cornacchia and P. Emma, Phys. Rev. ST Accel. Beams 5, 084001 (2002).

[8] P. Emma, Z. Huang, K.-J. Kim, and P. Piot, Phys. Rev. ST Accel. Beams 9, 100702 (2006).

[9] Y.-E Sun, P. Piot, A. Johnson, A. H. Lumpkin, T. J. Maxwell, J. Ruan, and R. Thurman-Keup, Phys. Rev. Lett. 105, 234801 (2010).

[10] J. Ruan, A. S. Johnson, A. H. Lumpkin, R. Thurman-Keup, H. Edwards, R. P. Fliller, T. Koeth, and Y.-E Sun, Phys. Rev. Lett. 106, 244801 (2011).

[11] Y.-E Sun, J. G. Power, K.-J. Kim, P. Piot, and M. Rihaoui, in Proceedings of the 2007 Particle Accelerator
Conference, Albuquerque, New Mexico (IEEE, Albuquerque, New Mexico, 2007), p. 3441.

[12] D. Xiang, Phys. Rev. ST Accel. Beams 13, 010701 (2010).

[13] R. Fliller, Fermilab Report No. BeamDocs 2553-v2, 2007.

[14] W. Wan (unpublished).

[15] A. Zholents and M. Zolotorev, New type of a bunch compressor and generation of a short wave length coherent radiation, LBNL CBP Seminar, 2010; see also Argonne National Laboratory Report No. ANL/APS/LS-327, 2011.

[16] J. Wang and S. Tantawi, in Proceedings of LINCA08, Victoria, Canada (2008), p. 966.

[17] SLAC Report No. SLAC-Report-411, 1993.

[18] D. Xiang, E. Colby, M. Dunning, S. Gilevich, C. Hast, K. Jobe, D. McCormick, J. Nelson, T. O. Raubenheimer, K. Soong, G. Stupakov, Z. Szalata, D. Walz, S. Weathersby, M. Woodley, and P-L. Pernet, Phys. Rev. Lett. 105, 114801 (2010).

[19] M. Borland, Advanced Photon Source Report No. LS-287, 2000.

[20] P. Piot, Y.-E Sun, J. G. Power, and M. Rihaoui, Phys. Rev. ST Accel. Beams 14, 022801 (2011).

[21] W.D. Kimura, A. van Steenbergen, M. Babzien, I. Ben-Zvi, L. P. Campbell, D. B. Cline, C. E. Dilley, J. C. Gallardo, S. C. Gottschalk, P. He, K. P. Kusche, Y. Liu, R. H. Pantell, I. V. Pogorelsky, D. C. Quimby, J. Skaritka, L. C. Steinhauer, and V. Yakimenko, Phys. Rev. Lett. 86, 4041 (2001).

[22] S. J. Russell, K. A. Bishofberger, B.E. Carlsten, D. C. Nguyen, and E. I. Smirnova, in Proceedings of the 23rd Particle Accelerator Conference, Vancouver, Canada, 2009 (IEEE, Piscataway, NJ, 2009), TH5PFP036.

[23] D. Xiang and Y. Ding, Phys. Rev. ST Accel. Beams 13, 094001 (2010).

[24] V. Yakimenko, M. Fedurin, V.N. Litvinenko, A. V. Fedotov, D. Kayran, and P. Muggli, in Proceedings of the 2011 Particle Accelerator Conference, New York (2011), WEP107. 